Journal of Animal and Veterinary Advances $10(4): 421-427,2011$

ISSN: $1680-5593$

(C) Medwell Journals, 2011

\title{
A Novel Pig Gene, PFKFB1, Differentially Expressed in the Muscle Tissues from Wujin Pigs and Large White Pigs
}

\author{
Liu Yonggang \\ College of Animal Science and Technology, \\ Yunnan Agricultural University, 650201 Kunming, China
}

\begin{abstract}
The mRNA differential display technique was performed to investigate gene expression differences in the longissimus dorsi muscle from Wujin and Large white pigs. A fragment of one differentially expressed gene was isolated and sequenced. A complete cDNA sequence of the gene was obtained using the Rapid Amplification of Cdna Ends (RACE) method. The open reading frame of this gene encodes a protein of 428 amino acids which is homologous with the 6-Phosphofructo-2-Kinase/Fructose-2,6-Biphosphatase 1 (PFKFB1) of 6 species: cattle (97\%), human (97\%), horse (96\%), rhesus monkey (96\%), rat (94\%) and mouse (94\%). This newly identified gene was respectively defined as the swine PFKFB1 gene and had been assigned GeneID: 100233197. The phylogenetic tree analysis revealed that the swine PFKFB1 gene has a closer genetic relationship with the PFKFB1 gene of cattle. The tissue expression analysis indicated that the swine PFKFB1 gene has a broad tissue distribution. The experiment is the first to establish the primary foundation for further research on the swine PFKFBl gene.
\end{abstract}

Key words: Pig, PFKFB1, muscle tissue, mRNA differential display, gene, China

\section{INTRODUCTION}

The mRNA differential display first described by Liang and Pardee (1992) remains an efficient tool for comparative profiling of gene expression under different experimental conditions. It has statistically been shown that 80-120 primer combinations would be sufficient to cover all the transcript populations in the cell (Liang et al., 1993). This assay possesses the following advantages: it is based on well established methods, $>2$ samples can be compared simultaneously and only a small amount of starting material is needed (Yamazaki and Saito, 2002).

Chinese indigenous pig breeds such as Wujin, Meishan, Erhualian and Tongcheng often have conspicuous flaws such as superabundant fat and too low lean meat rate while exotic pig breeds such as Large white, Landrace and Duroc always have lower fat and higher lean meat rates. Therefore, Chinese indigenous pigs are always named fat-type pigs while exotic pigs are always named the lean-type (Pan et al., 2003). Given that phenotypic variances are mainly determined by the genetic differences, the identification of differentially expressed genes between Chinese indigenous and exotic pig breeds can give considerable promises for breeding.

The present study was carried out with the mRNA differential display technique to identify the differentially expressed genes in the muscle tissues from one Chinese indigenous breed (Wujin) and one exotic pig breed (Large white). We provide here the results on the identification of previously unrecognised porcine gene, PFKFB1 which is differentially expressed in Large white versus Wujin skeletal muscle tissues.

\section{MATERIALS AND METHODS}

Sample collection: The longissimus dorsi muscle samples were collected from 120 days old Large white ( 5 males and 5 females) and Wujin ( 5 males and 5 females) pigs for mRNA differential display and semi-quantitative ReverseTranscription Polymerase Chain Reaction (RT-PCR) analyses.

The tissues including spleen, ovary, heart, small intestine, liver, lung, kidney, muscle and fat were collected from one adult Wujin $\mathrm{x}$ Large white cross pig for the later tissue expression profile analysis. Tissues were immediately frozen in liquid nitrogen and stored at $-80^{\circ} \mathrm{C}$. The total RNA was extracted from tissues using the total RNA extraction kit (Gibco, Grand Island, NY, USA) in accordance with the manufacturer's recommendations. Before the first-strand complementary DNA (cDNA) synthesis, DNase I treatment of the total RNA was done.

Differential display: The differential display PCR amplification of each reverse transcription product was carried out with 10 arbitrary and nine oligo (dT) primers as described by Liu et al. (2004, 2005). The PCR products 
were then separated on the $8 \%$ non-denaturing polyacrylamide gel and displayed using the silver stain (Liu et al., 2004, 2005).

Semi-quantitative RT-PCR: Semi-quantitative RT-PCR was performed as described by Liu (2009) and Liu and Xiong $(2009 \mathrm{a}, \mathrm{b})$. To avoid the influence of cDNA concentration on semi-quantitative RT-PCR, we repeated PCR amplifications using 100-500 ng cDNA as template. We selected the housekeeping gene GAPDH as the internal control. The control gene primers used were: 5'-ACCACAGTCCATGCCATCAC-3' (forward primer 1) and 5'- TCCACCACCCTGTTGCTGT -3' (reverse primer 1). The 420 bp PCR product was verified by sequencing. The following primers were used to perform the RT-PCR for identification and tissue expression profile analysis of the swine PFKFB1 gene: 5'-CAGTCCCTGCC CACTACT-3' (forward primer 2) and 5'-AGCTCCTTGGTT GTAGCTAG$3^{\prime}$ (reverse primer 2).

The PCR product was 208-bp in length (verified by sequencing). The $25 \mu \mathrm{L}$ reaction system contains $2 \mu \mathrm{L}$ cDNA (100-500 ng), 5 pmoles each oligonucleotide primer (forward primer 1 and 2, reverse primer 1 and 2), $2.5 \mu \mathrm{L}$ $2 \mathrm{mmol} \mathrm{L}^{-1}$ mixed dNTPs, $2.5 \mu \mathrm{L} 10 \times$ Taq DNA polymerase buffer, $2.5 \mu \mathrm{L} 25 \mathrm{mmol} \mathrm{L}^{-1} \mathrm{MgCl}_{2}$ and 2 units of Taq DNA polymerase. The PCR program initially started with a $94^{\circ} \mathrm{C}$ denaturation for $4 \mathrm{~min}$ followed by 25 cycles of 94,60 and $72^{\circ} \mathrm{C} / 50 \mathrm{sec}$ then $72^{\circ} \mathrm{C}$ extension for $10 \mathrm{~min}$, finally $4^{\circ} \mathrm{C}$ to terminate the reaction. PCR products were analysed in the linear range of amplification by agarose gel electrophoresis and intensity of bands was estimated using Glyco BandScan software (PROZYME $^{\circledR}$, San Leandro, CA, USA). The ratio of PFKFB1 to GAPDH was calculated using Excel program. Difference significance of ratios of PFKFB1 to GAPDH was analyzed with the Least square method (GLM procedure, SAS version 8.0).

Rapid amplification of cDNA ends ( 5 '- and 3 '-RACE): 5'- and $3^{\prime}$-RACE were performed as the instructions of BD SMART $^{\mathrm{TM}}$ RACE cDNA amplification kit (BD science, USA). The Gene-Specific Primers (GSPs) were: 5'-RACE GSP: 5'-GAAGCAGGTCTCTTCCTGTGAGGCC-3', 3'-RACE GSP:5'-CTCTGGACTTGGCCTCACAGGAAGA-3'.RACE touchdown PCRs were carried out with 5 cycles of $94^{\circ} \mathrm{C} / 30 \mathrm{sec}$ and $72^{\circ} \mathrm{C} / 3 \mathrm{~min}$ followed by 5 cycles of $94^{\circ} \mathrm{C} / 30 \mathrm{sec}, 70^{\circ} \mathrm{C} / 30 \mathrm{sec}$ and $72^{\circ} \mathrm{C} / 3 \mathrm{~min}$, finally with 30 cycles of $94^{\circ} \mathrm{C} / 30 \mathrm{sec}, 67^{\circ} \mathrm{C} / 30 \mathrm{sec}, 72^{\circ} \mathrm{C} / 3 \mathrm{~min}$ to terminate reaction.

The RACE PCR products were then cloned into pMD18-T vector (TaKaRa, Dalian, China) and sequenced bidirectionally with the commercial fluorometric method (Shenggong, Shanghai, China). At least five independent clones were sequenced for each PCR product.
Sequence analysis: The cDNA sequence prediction was conducted using GenScan software. Protein sequence prediction and analysis were performed using the conserved domain architecture retrieval tool of BLAST at the National Centre for Biotechnology Information (NCBI) server (http://www.ncbi.nlm.nih.gov/BLAST) and Clustal W software (http:/www.ebi.ac.uk/clustalw).

\section{RESULTS AND DISCUSSION}

mRNA differential display: From the mRNA differential display, one band, nominated as the band 162, later identified as a fragment of the PFKFB1 gene was found to be predominantly expressed in the longissimus dorsi muscle of Large white pigs while it was barely visible in the band pattern of the longissimus dorsi of Wujin pigs (Fig. 1).

Semi-quantitative RT-PCR: The differentially expressed band was recovered from gel and used as the template for the re-amplification which was performed with the corresponding oligo (dT) and arbitrary primers used in the mRNA differential display assay. The resulting PCR product was $313 \mathrm{bp}$ that was consistent with that of the differential display (Fig. 1). The purified PCR product was then cloned into the pMD18-T vector and the recombinant plasmid was sequenced. Semi-quantitative RT-PCR was conducted and the results (Fig. 2) indicated

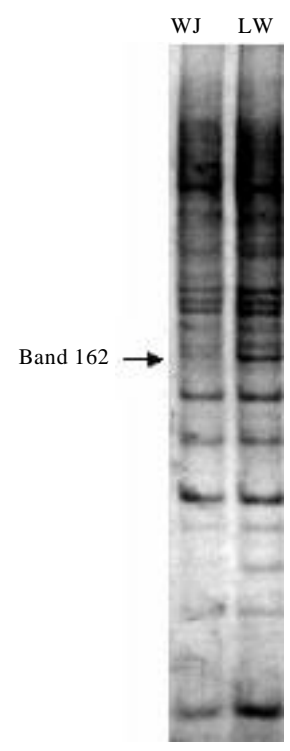

Fig. 1: Representative band pattern on mRNA differential display analysis showing upregulated band 162 (arrow, about $300 \mathrm{bp}$ ) in Large White (LW) vs Wujin (WJ) longissimus dorsi muscles 

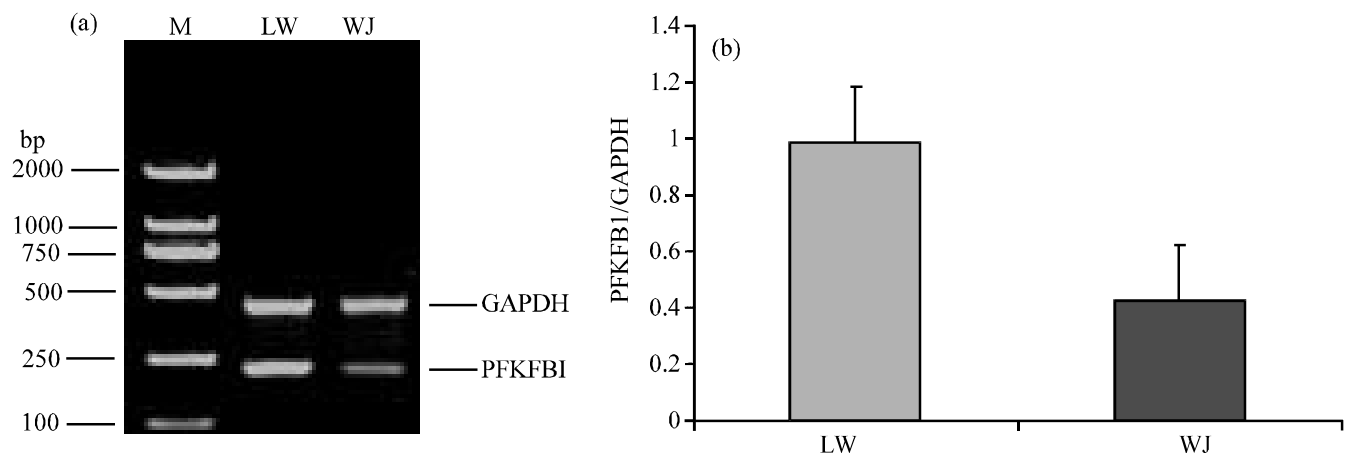

Fig. 2: (a) Representative and (b) calculated PFKFB1 mRNA expression levels in Large White (LW) vs Wujin (WJ) longissimus dorsi muscle samples (RT-PCR, $\mathrm{n}=5$ )

ACCCAAGCCCCCACTGGTAGATACAGGAGCAGAGGCAGGCAAGGCAGGCAGGACTAGGAGA GAGTAGAAGGGCTGGAGACAGGCTGCCAGAGGGCTCAGAACCCCAGCGCACCTCCCTGTCCC AACTCTGTCAACCTCCTGCTGTGGCCACTGCAACAGAAGAGACAGCTAATAAGACAGGAAGT GAGGCCTGGTACCTTGTGGACAGTGGTGTCATTAGCTGCGACGCCTAAGATGACTCAAGAGA TGGGAGAGCTCACCCAAACC MTQEMGELTQT

AGATTGCAGAAGATCTGGATTCCACATAGCAGCGGCAACAGTAGGCTG CAACGGAGGAGGGGCTCA RLQKIWIPHSSGNSRLQRRRGS TCCATACCCCAGTTTACAAATTCCCCCACGATGGTGATTATGGTGGGTT TACCAGCTCGAGGAAAG SIPQFTNSPTMVIMVGLPARGK ACCTACATCTCCACGAAGCTCACACGTTATCTCAATTGGATAGGAACAC CAACTAAAGTGTTTAAT TYISTKLTRYLNWIGTPTKVFN TTAGGCCAGTATCGACGAGAGGCAGTGAGCTACAAGAACTACGAATTC TTTCTCCCAGACAACATG LGQYRREAVSYKNYEFFLPDNM GAGGCCCTACTTATAAGGAAGCAGTGTGCCCTGGCAGCTCTGAAAGAT GTCCATAACTATCTTAGC

EALLIRKQCALAALKDVHNYLS CATGAGGAAGGTCACGTTGCGGTTTTTGATGCCACCAATACTACCAGAG AAAGACGGTCTTTGATT HEEGHVAVFDATNTTRERRSLI CTACAGTTTGCTAAAGAACACGGTTATAAGGTCTTTTTCATTGAGTCCA TTTGTAATGACCCCGAC LQFAKEHGYKVFFIESICNDPD GTCATTGCAGAAAACATCAGGCAAGTGAAGCTTGGCAGCCCTGATTAT ATAGACTGTGACCGTGAA VIAENIRQVKLGSPDYIDCDRE AAGGTTCTAGAAGACTTTCTAAAAAGAATCCAGTGCTATGAGGTCAAC TACCAACCTTTGGATGAT KVLEDFLKRIQCYEVNYQPLDD GAACTGGACAGCCACTTGTCCTACATCAAGATCTTCGACGTGGGCACAC GCTACATGGTGAACCGC

Fig. 3: Continued 
J. Anim. Vet. Adv., 10 (4): 421-427, 2011

\author{
ELDSHLSYIKIFDVGTRYMVNR \\ GTGCAGGACCACATCCAGAGCCGCACAGTCTACTATCTCATGAACATCCATGTCACACCCCGCTCC \\ VQDHIQSRTVYYLMNIHVTPRS \\ ATCTACCTATGCCGGCACGGTGAGAGTGAACTCAACCTCAGAGGCCGCATCGGAGGTGACTCTGGC \\ IYLCRHGESELNLRGRIGGDSG \\ CTCTCACCTCGGGGCAAGCAGTATGCCTATGCCTTGGCCGACTTCATTAAGTCCCAGGCCATCAGC \\ LSPRGKQYAYALADFIKSQAIS \\ TCCCTGAAGGTGTGGACCAGCCACATGAAGAGGACTATCCAGACAGCTGAAGCCCTGGATGTCCCC \\ SLKVWTSHMKRTIQTAEALDVP \\ TATGAGCAGTGGAAGGCCCTGAATGAGATTGATGCGGGTGTCTGTGAAGAAATGACATATGAGGAA \\ YEQWKALNEIDAGVCEEMTYEE \\ ATCCAAGAGCACTATCCCGAAGAATTTGCCCTACGAGACCAAGATAAATATCGCTACCGCTATCCC \\ IQEHYPEEFALRDQDKYRYRYP \\ AAGGGAGAGTCCTATGAAGATCTGGTCCAGCGTCTGGAGCCAGTTATAATGGAGCTAGAACGTCAG \\ KGESYEDLVQRLEPVIMELERQ \\ GAAAATGTATTAGTGATTTGCCACCAGGCTGTCATGCGGTGCCTCCTGGCCTACTTCCTGGACAAG \\ ENVLVICHQAVMRCLLAYFLDK \\ AGCTCAGATGAGCTGCCATATCTCAAGTGCCCTCTGCACACAGTGCTCAAACTTACGCCTGTGGCT \\ SSDELPYLKCPLHTVLKLTPVA \\ TATGGCTGCAAAGTGGAGTCGATCTACCTGAATGTGGAGGCTGTCAACACACACCGGGAGAAGCCT \\ YGCKVESIYLNVEAVNTHREKP \\ GAGAATGTAGACATCACCCGAGAACCTGAGGAAGCTCTGGACACAGTCCCTGCCCACTACTGAGCAC \\ ENVDITREPEEALDTVPAHY? \\ TTTCTAAGACATCAAACTTCCTCTGTCCTAGCTCTCTTCCAACTTTAGGAGGTGA \\ CGTCATTGTTCTCCTACCCTGAGAATACTCTGGACTTGGCCTCACAGGAAGAGAC \\ CTGCTTCCAGTGAAGAAACTCTCATCAGCTCTGAAACAAGTCTTGACGTCTAGCT \\ ACAACCAAGGAGCTATCTAGCTCAGGAAGAAACTTTTTCTTTCTTAATTCCTATT \\ CCCTAGTCAATAAAGACTTCTGTTACTGACCAAAAAAAAAAA
}

Fig. 3: The cDNA and amino acid sequence of the swine gene containing band 162 (GenBank accession number: FJ436397). ATG, start codon; TGA, stop codon (* -stop codon)

that the band 162 (PFKFB1) is predominantly expressed in the longissimus dorsi muscle of Large white pigs. Through, 5'-RACE, one PCR product of 1771 bp was obtained. The 3'-RACE product was $180 \mathrm{bp}$. These products were then cloned to t-vector and sequenced. The alignment of these $35 \mathrm{bp}$ overlapping sequences yielded a 1916 bp cDNA sequence (Fig. 3). The nucleotide analysis using the BLAST software at NCBI server (http:/www.ncbi.nlm.nih.gov/BLAST), revealed that a 1916 bp cDNA sequence was not homologous to any of the known porcine genes and it was then deposited into the GenBank database (Accession number: FJ436397). The sequence prediction was carried out using the GenScan software.

An Open Reading Frame (ORF) encoding 428 amino acids was found in the 1916-bp cDNA sequence. A probability of exon was 0.777 , poly-A signal was from 1883-1888 bp (consensus: AATAAA). The complete cDNA sequence of this gene and the encoded amino acids are shown in Fig. 3.
Further BLAST analysis revealed that the protein sequence is characterized by a high homology with that of the 6-Phosphofructo-2-Kinase/ Fructose-2,6-Biphosphatase 1 (PFKFB1) of cattle (Accession number: NP 776997; 97\%), human (Accession number: NP 002616; 97\%), horse (Accession number: XP_001494106; 96\%), rhesus monkey (Accession number: XP_001091907; 96\%), rat (accession number: NP_036753; 94\%), mouse (accession number: P70266; 94\%) (Fig. 4).

On the basis of results obtained in the experiments we assume that this gene can be defined as the swine PFKFBl gene. Based on the results of the alignment of six known PFKFB1 proteins, a phylogenetic tree was constructed using the Clustal W software (Fig. 5).

The swine PFKFB1 gene has a closer genetic relationship with the cattle PFKFB1 gene than with human, horse, rhesus monkey, rat and mouse ones. 
Pig

Cattle

Horse

Human

Rhesus monkey

Rat

Mouse

Pig

Cattle

Horse

Human

Rhesus monkey

Rat

Mouse

Pig

Cattle

Horse

Human

Rhesus monkey

Rat

Mouse

Pig

Cattle

Horse

Human

Rhesus monkey

Rat

Mouse

Pig

Cattle

Horse

Human

Rhesus monkey

Rat

Mouse

Pig

Cattle

Horse

Human

Rhesus monkey

Rat

Mouse

Pig

Cattle

Horse

Human

Rhesus monkey

Rat

Mouse

Pig

Cattle

Horse

Human

Rhesus monkey

Rat

Mouse
MTQEMGE LTQTRLQKIWI PHSSGNSRLQRRRGSSIPQFTNSPTMVIMVGL PARGKTYIST MSQEMGE LTQTRL QKIUI PHNNGNSRL QRRRGSS I PQFTNS PTMVIMVGL PARGKTYIST MSQEMGE LTQTRLQKIWI PHSSGSSGLQRRRGSSIRQFTNSPTMVIMVGL PARGKTYIST MSPEMGE LTQTRLQKIUI PHSSGSSRLQRRRGSSIPQFTNSPTMVIMVGL PARGKTYIST MS PEMGE LTQTRLQKIWI PHSSGSSRLHRRRGSS I PQFTNS PTMV IMVGL PARGKTYIST MSREMGE LTQTRLQKIUI PHSS SSVLQRRRGSSI PQFTNS PTMVIMVGL PARGKTYIST MSREMGE LTQTRLQKIWI PHSS SS L LQRRRGSS I PQFTNSPTMVIMVGL PARGKTYIST

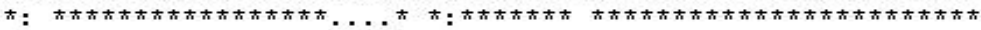

KLTRYLNJIGTPTKVFNL GQYRREAVSYKNYE FFL PDNMEAL L IRKQCÄLÄALKDVHNYL KLTRYLNUI GTPTKVFNL GQYRREAVSYKNYE FFL PDNMEAL L IRKQCALAAALKDVHSYL KLTRY LNJI I TTPTKV FNL GQYRREAVSYKNYE FFL PDMMEAL L IRKQCALAAALKDVHDYL KLTRYLNNI GTPTKVFNL GQYRREAVSYKNYE FFL PDNMEAL Q IRKQCALAALLKDVHNYL KLTRYLNJIGTPTKVFNL GQYRREAVSYKNYE FFL PDNMEAL QIRKQCALAALLKDVHNYL KLTRYLNJI GTPTKVFNL GQYRREAVSYRNYE FFRPDNTEA A L IRKQCALAAALKDVHKYL KLTRYLNJI GTPTKVFNL GQYRREAVSYRNYE FFR PDNMEAOL IRKQCALAALKDVHKYL 年

SHEE GHVAVFDATNTTRERRS L I LQFAKEHGYKVFFIESICND PDVIAENIRQVKLGSPD SHEE GRVAVFDATNTTRERRS L I L FAKEHGYKVFFIE SICND PDVIAENIRQVKLGSPD SHEE GHVAVFDATNTTRERRS L I LQFAKEHGYKVFFIE SICND P GI IAENIRQVKLGSPD SHEE GHVAVFDATNTTRERRS L I LQFAKEHGYKVFFIESICND PGI IAENIRQVKLGSPD SREE GHVAVFDATNTTRERRS LI L QFAKEHGYKVFFIE S ICND P GVIAENIRQVKLGSPD SREE GHVAVFDATNTTRERRS L I LQFAKEHGYKVFFIE SICND PE I IAENIKOVKL GSPD SREE GHVAVFDATNTTRERRS L I Q QFAKEHGYKVFFIE SICND PDI IAENIKQVKLGSPD

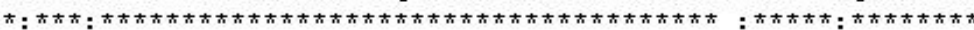

YIDCDREKVLED FLKRIQCYEVNYQP LDDE LDSHLSYIKI FDVGTRYMVRVQDHIQSRT YIDCDREKVLED FLKRIECYEVNYQP LDDE LDSHL SY IKI FDVGTRYMVNRVQDHIQSRT YIDCDREKVLED FLKR IE CYKVNYQP LDDE LDSHLSYIKI FDVGTRYMVNRVQDHIQSRT YIDCDREKVLED FLKRIECYEVNYQP LDEE LD SHLSYIKI FDVGTRYMNRVQDHIQSRT YVDCDREKVLED FLKR IECYEVNYQP LDEE LDSHLSYIKI FDVGTRYMVNRVQGHIQSRT YIDCD QEKVLED FLKR IE CYE INYQP LDEE LDSHL SYIKI FDVGTRYMVNRVQDHVQSRT YIDCDQEKVLED FLKRIECYE INYQP LDEE LD SHLSYIKI FDVGTRYMNNRVDHVQSRT

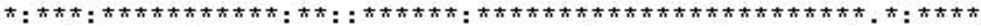

VYYLMNIHVTPRSIYLCRHGESELNLRGRIGGDSGLSPRGKOYAYALADFIKSQÄISSLK VYYLMNIHVTPRSIYLCRHGESE LNLRGRIGGDSGLSARGKOYAYALANFIQSQGISSLK VYYLMNIHVTPRSIYLCRHGE SE LNLRGRIGGDSGLSARGKQYAYALANFIQSQGISSLK WYLMNIHVTPRSIYLCRHGESE LNIRGR IGGD SGLSVRGKOYAYALANFIQSQGISSLK VYYLMIHVTPRSIYLCRHGE SD LNLRGRIGGDSGLSVRGKQYAYALANFIQSQGISSLK AYYLMNIHVTPRS IYLCRHGE SE LNLRGRI GGD SGLSARGKOYAYALANFIRSQGISSLK AYYLMNIHVTPRSIYLCRHGE SE LNLRGRIGGDSGLSARGKQYAYALANFIRSQSISSLK

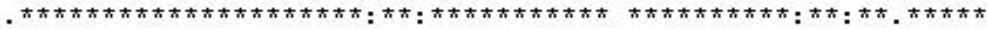

VWTSHMKRTIQTÄEALDVPYEQTKALNE IDAGVCEEMTYEE IQEHYPEE FALRDQDKYRY VGTSHMKRTIQTAEALGL PYE QUKALNE IDAGVCEEMTYEE I QEHY PEE FALRDQDKYRY VWTSHMIRRTIQTAEALGVPYE QWKALNE IDAGVCEEMTYEE I QEHYPEE FALRDQDKYRY VWTSHMKRTIQTAEALGVPYE QUKALLNE IDAGVCEEMTYEE IQEHY PEE FALRDQDKYRY VITSHMKRTIQTAEAL GVPYE QUKALNE IDAGVCEEMTYEE I QEHY PEE FALRDQDKYRY VUTSHMKRTIQTAEAL GVPYE QWKALLNE IDAGVCEEMTYEE IQEHY PEE FALRDQDKYRY VUTSHMKRTIQTAEAL GVPYE QUKALNE IDAGVCEEMTYEE IQEHY PEE FALRDQDKYRY

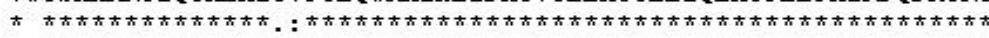

RYPKGE SYED LVQRLE PVIME LERQENVLVICHQAVMRC L LAYFLDKS SDE L PYLKC P LH RY PKGE SYED LVQRLE PVIME LERQENVLVICHQAVMRC L LAY FLDKS SDE L PYLKC P LH RYPKGE SYED LVQRLE PVIME LERQENVLVICHQAVMRC L LAY FLDKSSDE L PYLKC P LH RYPKGESYED LVQRLE PVIME LERQENV LVICHQAVMRC L LAY FLDKS SDE L PYLKC P LH RYPKGESYED LVQRLE PVIME LERQENVLVICHQAVMRC L LAY FLDKSSDE L PYLKC P LH RYPKGE SYED L VQRLE PVIME LERQENVLVICHQAVMRC L LAY FLDKS SDE L PYLKC P LH RYPKGE SYED LVQRLE PVIME LERQENVLVICHQAVMRC L LAY FLDKS SDE L PYLKC P LH

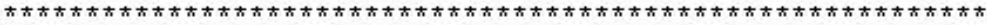

TVLKLTPVAYGCKVE S IYLNVEAVNTHREKPENVD ITRE PEEALDTVPAHY TVLKLTPVAYGCKVE S IYLNVEAVNTHREKPENVD ITRE PEEALDTVPAHY TVLKLTPVAYGCKVE SIYLNVEAVNTHREKPENVDITRE PEEALDTVPDHY TVLKLTPVAYGCKVE S I YLNVEAVNTHREKPENVD ITRE PEEALDTVPAHY TVLKLTPVAYGCKVE S IYLNVEAVNTHREKPENVD ITRE PEEALDTVPAHY TVLKLTPVAYGCRVE S I YLNVEAVNTHRDKPENVD ITREAEEALDTVPAHY TVLKLTPVAYGCRVE S IYLNVEAVNTHRDKPENVD ITRE PEEALD TVPAHY

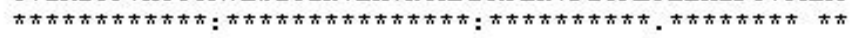

Fig. 4: The alignment of PFKFB1 proteins 


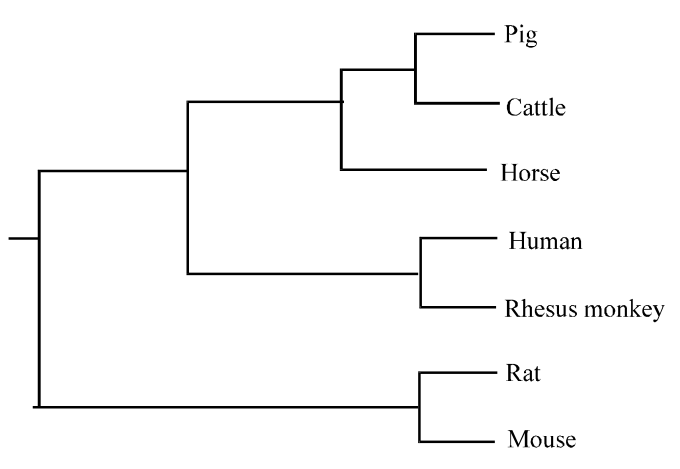

Fig. 5: The phylogenetic tree for the PFKFBI genes

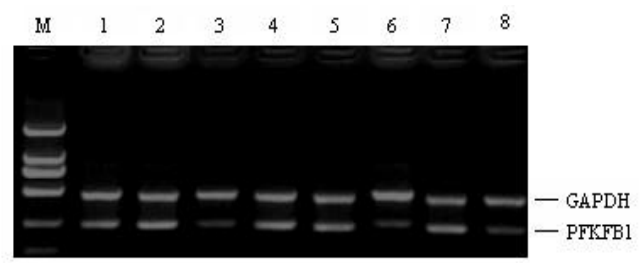

Fig. 6: RT-PCR profiling of the swine PFKFB1 gene expression. M, DL2000 marker kit (marker size as same as the Fig.1); 1, liver; 2, muscle; 3, ovary; 4, small intestine; 5 , heart; 6 , fat; 7 , lung; 8 , kidney; 9, spleen

Tissue expression: The RT-PCR profiling of tissue expression of the swine PFKFB1 gene was carried out using samples from one adult Wujin $\mathrm{x}$ Large white cross pig. The swine PFKFB1 gene is predominantly expressed in muscle, heart, lung, liver, small intestine and kidney and weakly expressed in fat, ovary and spleen (Fig. 6).

PFKFB1, this gene encodes a member of the family of bifunctional 6-phosphofructo-2-kinase:fructose-2,6-biphosphatase enzymes. The enzyme forms a homodimer that catalyzes both the synthesis and degradation of fructose-2,6-biphosphate using independent catalytic domains. Fructose-2,6-biphosphate is an activator of the glycolysis pathway and an inhibitor of the gluconeogenesis pathway. Consequently, regulating fructose-2,6-biphosphate levels through the activity of this enzyme is thought to regulate glucose homeostasis (Algaier and Uyeda, 1988; Lee et al., 2003; Veech, 2003). To this date, the PFKFB1 gene was indentified and characterized in horse, dog, human, olive baboon, red-bellied titi and other animals; the swine PFKFB1 has not been reported yet.

\section{CONCLUSION}

The present results show that the PFKFB1 gene is differentially expressed in the longissimus dorsi muscle being a more abundant in Large white than in Wujin pigs. Wujin is a fat type pig breed comprising much more body fat than lean meat/muscle. On the other hand, Large white is a typical lean type pig breed, presenting the opposite phenotype to that described for the Wujin breed. The two pig breeds used in this study, Large white and Wujin, differ in lean meat percentage.

It is therefore, interesting that the expression of the swine PFKFB1 gene in the longissimus dorsi muscle shows the trend of a higher expression in Large white as compared with Wujin

A major question is the extent to which such predominant expression could be developmentally or metabolically significant in terms of acquiring of any phenotypic change in favor of a higher lean-type rate. Clearly, this merits needs further study.

\section{ACKNOWLEDGEMENT}

This researchers was supported by the National Natural Science Foundation of China (No. 30800810).

\section{REFERENCES}

Algaier, J. and K. Uyeda, 1988. Molecular cloning, sequence analysis, and expression of a human liver cDNA coding for fructose-6-P,2-kinase: Fructose-2,6bisphosphatase. Biochem. Biophys. Res. Commun., 153: 328-333.

Lee, Y.H., Y. Li, K. Uyeda and C.A. Hasemann, 2003. Tissue-specific structure/function differentiation of the liver isoform of 6-phosphofructo-2kinase/fructose-2,6-bisphosphatase. J. Biol. Chem., 278: 523-530.

Liang, P. and A.B. Pardee, 1992. Differential display of eukaryotic messenger RNA by means of the polymerase chain reaction. Science, 257: 967-971.

Liang, P., L. Averboukh and A.B. Pardee, 1993. Distribution and cloning of eukaryotic mRNAs by means of differential display: Refinements and optimization. Nucl. Acids Res., 21: 3269-3275.

Liu, G.Y. and Y.Z. Xiong, 2009a. Molecular characterization and expression profile of a novel porcine gene differentially expressed in the muscle tissues from Meishan, Large White and their hybrids. Mol. Biol. Rep., 36: 57-62.

Liu, G.Y. and Y.Z. Xiong, 2009b. Molecular cloning, polymorphism and association analyses of a novel porcine mRNA differentially expressed in the Longissimus muscle tissues from Meishan and Large White pigs. Mol. Biol. Rep., 36: 1393-1398.

Liu, G.Y., 2009. cDNA cloning, sequence identification and tissue expression distribution of three novel porcine genes: UCHL3, RIT1 and CCND3. Mol. Biol. Rep., 36: 521-528. 
Liu, G.Y., Y.Z. Xiong and C.Y. Deng, 2005. Isolation, identification of differentially expressed sequence tags in the backfat tissue from Meishan, Large White and Meishan x Large White cross pigs. Agric. Sci. China, 4: 101-105.

Liu, G.Y., Y.Z. Xiong, C.Y. Deng, B. Zuo and J.H. Zhang, 2004. Comparison of gene expression patterns in Longissimus dorsi of pigs between the high-parent heterosis cross combination Landrace $\mathrm{x}$ Large White and the mid-parent heterosis cross combination Large White x Meishan. Asian-Aust. J. Anim. Sci., 17: 1192-1196.
Pan, P.W., S.H. Zhao, M. Yu, T.A. Xiong and K. Li, 2003. Identification of differentially expressed genes in the Longissimus dorsi dorsi tissue between Duroc and Erhualian pigs by mRNA differential display. AsianAust. J. Anim. Sci, 16: 1066-1070.

Veech, R.L., 2003. A humble hexose monophosphate pathway metabolite regulates short- and long-term control of lipogenesis. Proc. Natl. Acad. Sci. USA., 100: $5578-5580$.

Yamazaki, M. and K. Saito, 2002. Differential display analysis of gene expression in plants. Cell. Mol. Life Sci., 59: 1246-1255. 